\title{
IL-17 is a potential biomarker for predicting the severity and outcomes of pulmonary contusion in trauma patients
}

\author{
SHILAI LI ${ }^{1}$, QUANLIN QIN ${ }^{1}$, DAQING LUO ${ }^{1}$, WENHUI PAN ${ }^{1}$, YUQING WEI ${ }^{1}$, YANSONG XU ${ }^{1}$, \\ JUNXUAN WANG $^{2}$, XINPING YE ${ }^{3}$, JIJIN ZHU ${ }^{1}$ and LIMING SHANG ${ }^{3}$ \\ Departments of ${ }^{1}$ Emergency, ${ }^{2}$ Medical Records and ${ }^{3}$ Hepatobiliary Surgery, \\ The First Affiliated Hospital of Guangxi Medical University, Nanning, Guangxi 530021, P.R. China
}

Received August 14, 2020; Accepted October 16, 2020

DOI: $10.3892 /$ br.2020.1381

\begin{abstract}
Pulmonary contusion (PC) is very common in blunt chest trauma, and always results in negative pulmonary outcomes, such as pneumonia, acute respiratory distress syndrome (ARDS), respiratory failure or even death. However, there are no effective biomarkers which can be used to predict the outcomes in these patients. The present study aimed to determine the value of interleukin (IL)-17 and IL-22 in predicting the severity and outcomes of $\mathrm{PC}$ in trauma patients. All trauma patients admitted to The First Affiliated Hospital of Guangxi Medical University between January 2015 and December 2017, were studied. Patients aged $>14$ years old with a diagnosis of $\mathrm{PC}$ upon their admission to the emergency department were included. Patients with PC were enrolled as the PC group, patients without PC were enrolled as the non-PC group, and healthy individuals were selected as the control group. Clinical information, including sociodemographic parameters, clinical data, biological findings and therapeutic interventions were recorded for all patients who were enrolled. Blood samples were collected and stored according to the established protocols. PC volume was measured by computed tomography and plasma cytokine levels were assayed by ELISA. A total of 151 patients with PC (PC group) and 159 patients without $\mathrm{PC}$ (non-PC group) were included in the present study. In addition, 50 healthy individuals were used as the control group. The primary cause of PC was motor vehicle crashes. PC patients had more rib fractures, but similar injury severity scores compared with other patients. More patients
\end{abstract}

Correspondence to: Dr Jijin Zhu, Department of Emergency, The First Affiliated Hospital of Guangxi Medical University, 6 Shuangyong Road, Nanning, Guangxi 530021, P.R. China

E-mail: zhujijin63@vip.sina.com

Dr Liming Shang, Department of Hepatobiliary Surgery, The First Affiliated Hospital of Guangxi Medical University, 6 Shuangyong Road, Nanning, Guangxi 530021, P.R. China

E-mail: shangliming@gxmu.edu.cn

Key words: interleukin 17, pulmonary contusion, trauma, patient outcomes, complications received Pleurocan drainage treatment and had pneumonia complications in the PC group compared with the other two groups. PC patients had a high incidence of ARDS and admission to the intensive care unit (ICU). PC patients also experienced longer periods on mechanical ventilation and had longer stays in the ICU and hospital. PC volume was effective in predicting the outcomes of PC patients. IL-22 levels were similar in the PC group and non-PC group. However, IL-17 could be used as a biomarker to predict the severity of PC, and was strongly associated with PC volume. IL-17 was significantly associated with pro-inflammatory complications in PC patients and could be used as a biomarker for predicting in-patient outcomes of patients with PC. In conclusion, IL-17 is a potential biomarker for predicting the severity and outcomes of PC in trauma patients.

\section{Introduction}

Pulmonary contusion (PC) is the most commonly diagnosed intrathoracic injury resulting from chest blunt trauma, which is usually caused by a high-kinetic energy chest wall impact (1). It has been reported that the incidence of PC is $25-35 \%$ of all blunt chest traumas (1-3). Patients with PC are vulnerable to negative pulmonary outcomes, which include pneumonia, acute respiratory distress syndrome (ARDS), respiratory failure and death (4). The possible mechanisms are based on inertial effects, primarily on different tissue densities of light alveolar tissue and heavy hilar structures (2). Interstitial oedema and inflammatory cell infiltration following parenchymal injury result in lung hemorrhage, and PC develops slowly over the course of 24-48 h after chest injury. Supportive care, including mechanical ventilation, is the primary clinical management course for PC, and it is typically resolved within 3-14 days, depending on the size (1).

Interleukin (IL)-17 belongs to the IL-17 cytokine family and has a molecular weight of $35 \mathrm{kDa}$. IL-17 was initially shown to be secreted by a subset of $\mathrm{CD} 4^{+} \mathrm{T}$ cells, termed $\mathrm{Th} 17$ cells. These cells also secrete cytokines IL-22 and IL-21 (5). IL-17 primarily affects epithelial cells in tissues, such as the gut, lung and skin (5), and was found to serve a key role in host defense against infection, development of inflammatory diseases (6), and enhancement of mucosal barrier repair and maintenance by stimulating epithelial cell proliferation and 
tight junction protein production (5). It was reported that IL-17 is a useful biomarker for predicting the outcomes in patients who have suffered a physical trauma $(7,8)$. However, whether IL-17 is related to the severity and outcomes of PC remains unknown.

IL-22 belongs to the IL-10 cytokine family and is produced primarily by innate and adaptive T cells, such as Th22, Th17, and Th1 cells (9). It has the ability to strengthen homeostatic epithelial barrier functions in addition to the robustness and strength resistance of tissue (10). IL-22 has been reported to serve a critical role in maintaining epithelial integrity during pulmonary infection (11) and ventilator-induced lung injury (12). It has also been shown that IL-22 interacts with IL-17 to coordinate pulmonary immune defense during lung inflammation (13). Furthermore, the IL-17/IL-22 axis was found to serve important roles in burn injuries (14) and sepsis-induced lung injury (15). However, whether IL-22 is related to the severity and outcomes of PC remains unknown.

In the present study, the hypothesis that IL-17 and IL-22 may be used as predictors of the severity and outcomes of PC patients was assessed. The results showed that the severity of PC was a risk factor for in-patient outcomes, and IL-17, but not IL-22, could be used as a biomarker to predict the severity of PC. Furthermore, IL-17 could also be used as a biomarker for predicting the outcomes of in-patients who had suffered a PC.

\section{Materials and methods}

Patient enrolment. All trauma patients admitted to The First Affiliated Hospital of Guangxi Medical University between January 2015 and December 2017, were studied. All patients who met the following criteria were included: Age, $>14$ years old; patients with chest computed tomography (CT) scans and those diagnosed with PC at admission to the Department of Emergency, The First Affiliated Hospital of Guangxi Medical University. All patients lacking clinical or biological data and chest CT investigations were excluded. Clinical information, including sociodemographic parameters, clinical data, biological findings and therapeutic interventions, was recorded for all patients who were enrolled. The injury severity score (ISS) was measured to evaluate the seriousness of patients as previously described (16). Written informed consent was obtained from each participant prior to enrolment, and this study was approved by the Medical Ethics Committee of The First Affiliated Hospital of Guangxi Medical University.

During the present study period, 23,246 trauma patients were admitted to The First Affiliated Hospital of Guangxi Medical University. Of these, 438 patients (438/23 246, 1.9\%) had blunt chest trauma, of which 128 patients were excluded (53 patients lacking data, 51 patients lacking chest imaging investigation, 24 patients died shortly after admission to the hospital), and thus 310 patients were enrolled in the present study, 151 patients with PC (34.5\%, PC group) and 159 patients without PC (65.5\%, non-PC group). Additionally, 50 healthy individuals were enrolled as controls. A similar percentage of participants were male [PC group vs. non-PC group, $116(76.8 \%)$ vs. $115(72.3 \%)$ ], respectively $(\mathrm{P}=0.364)$, and mean ages were also similar in each group (PC group vs. non-PC group, mean \pm standard deviation, $42.2 \pm 15.9$ vs. $39.0 \pm 15.1$. The median age was 42 years old (range, $15-86$ years) in the
PC group vs. 37 years old (range, 15-89 years) in the non-PC group, and did not differ significantly $(\mathrm{P}=0.066$; Table I).

Blood samples. Whole-blood samples were taken within $2 \mathrm{~h}$ after admission, and then taken on days 3,5 and 7 after trauma. Blood samples were centrifuged at $500 \mathrm{x} \mathrm{g}$ for $10 \mathrm{~min}$ in a refrigerated $\left(4^{\circ} \mathrm{C}\right)$ centrifuge, and then the plasma was collected and stored at $-80^{\circ} \mathrm{C}$ until assayed. Blood samples were taken only once in the healthy group.

Pulmonary contusion volume measurement. The appearance of CT scan for PC shows nonspecific, focal, or diffuse alveolar infiltrates over the lung fields, and it always appears as areas of lung consolidation (Fig. 1). PC volume was measured as previously described (17). Briefly, trauma patients with suspected chest injuries were scanned with a 64-slice CT scanner (Siemens Healthcare $\mathrm{GmbH}$ ). The volume of lung contusion was measured by the $\mathrm{CT}$ volume, which was calculated based on the ratio of contused lung to the total lung volume. Images were evaluated by two consultant radiologists and 3-dimensional volumetric analysis of the data was calculated on the workstation (ReconCT version 13.8.2.0; Siemens Healthcare $\mathrm{GmbH}$ ). The total volume of lung contusions in both lungs was calculated and expressed as a percentage of the total lung volume.

ELISA. The plasma levels of IL-17 and IL-22 were measured by ELISA according to the manufacturer's protocol (IL-17, cat. no. D1700; IL-22, cat. no. D2200; R\&D Systems, Inc.).

Statistics. SPSS version 22.0 (IBM Corp.) was used to perform the statistical analyses. Differences in continuous variables are presented as the mean \pm standard deviation, and were calculated using a Student's t-test or a Mann-Whitney U test, whereas a $\chi^{2}$ test or Fisher's exact test was used to analyze categorical variables. Spearman's rank correlation coefficient analysis was performed to determine correlations. Factors independently associated with PC outcomes were determined by the binary logistical regression model. Receiver operating characteristic (ROC) curve analysis was performed to determine the predictive value of $\mathrm{PC}$ volume and IL-17 for PC outcomes. $\mathrm{P}<0.05$ was considered to indicate a statistically significant difference.

\section{Results}

Sociodemographic characteristics of patients with or without pulmonary contusion. There were no significant differences between preexisting comorbidity status (diabetes mellitus, hypertension and chronic obstructive pulmonary disease) or smoking history between the groups. There was a higher percentage of individuals who had experienced a motor vehicle crash in the PC group compared with the non-PC group [61 (40.4\%) vs. 44 (27.7\%), respectively; $\mathrm{P}=0.018$ ], whereas other causes, such as falling off a bicycle, falling down whilst walking/running, falling from a high place, or getting hit in the chest by an explosion, were similar between groups (Table I). For the control group, the male percentage of participants was $76.0 \%(38 / 50)$, and the mean age was $40.3 \pm 14.6$ years old. There were no significant differences in the sex distribution between any of the groups. 
Table I. Sociodemographic characteristics of the patients with or without pulmonary contusion.

\begin{tabular}{|c|c|c|c|}
\hline Characteristics & $\mathrm{PC}$ group, $\mathrm{n}=151^{\mathrm{a}}$ & Non-PC group, $n=159^{a}$ & $\mathrm{P}$-value \\
\hline Age, years & $42.2 \pm 15.9$ & $39.0 \pm 15.1$ & 0.066 \\
\hline Sex (male/female) & $116 / 35$ & $115 / 44$ & 0.364 \\
\hline Mechanism of injury & & & $<0.001^{\mathrm{b}}$ \\
\hline Motor vehicle crash & 61 & 44 & $0.018^{\mathrm{b}}$ \\
\hline Bicycle & 22 & 21 & \\
\hline Pedestrian & 10 & 35 & \\
\hline Fall & 39 & 25 & \\
\hline Explosion & 19 & 34 & \\
\hline Comorbidities & & & 0.637 \\
\hline Diabetes mellitus & 10 & 13 & \\
\hline Hypertension & 15 & 11 & \\
\hline Smoking & 20 & 27 & \\
\hline COPD & 5 & 8 & \\
\hline None & 101 & 100 & \\
\hline \multicolumn{4}{|l|}{ Localization } \\
\hline Right & 23 & - & \\
\hline Left & 18 & - & \\
\hline Bilateral & 110 & - & \\
\hline \multicolumn{4}{|l|}{ Other types of chest trauma } \\
\hline None & 84 & 126 & $<0.001^{\mathrm{b}}$ \\
\hline $\begin{array}{l}\text { Pneumothorax/Haemothorax/ } \\
\text { Haemopneumothorax/Rib fractures }\end{array}$ & $5 / 13 / 23 / 26$ & $7 / 7 / 5 / 14$ & \\
\hline Rib fractures, $\leq 3 ;>3$ & $115 / 36$ & $157 / 2$ & $<0.001^{\mathrm{b}}$ \\
\hline ISS & $31.9 \pm 10.0$ & $29.9 \pm 9.5$ & 0.071 \\
\hline ISS,$\leq 16 ;>16$ & $15 / 136$ & $25 / 134$ & 0.129 \\
\hline Polytrauma, yes/no & $129 / 22$ & $120 / 39$ & $0.027^{\mathrm{b}}$ \\
\hline $\mathrm{SaO}_{2}, \%$ & $98.8 \pm 2.9$ & $98.4 \pm 3.4$ & 0.324 \\
\hline Thoracic trauma treatment & & & $<0.001$ \\
\hline Conservative & 110 & 146 & \\
\hline Pleurocan drainage & 41 & 13 & \\
\hline Complications & & & $<0.001$ \\
\hline None & 56 & 140 & \\
\hline Pneumonia & 49 & 14 & \\
\hline Pleural effusion & 26 & 5 & \\
\hline Pneumonia and pleural effusion & 20 & 0 & \\
\hline ARDS, yes/no & $28 / 123$ & $12 / 147$ & $0.004^{\mathrm{c}}$ \\
\hline Blood products, yes/no & $32 / 119$ & $27 / 132$ & 0.345 \\
\hline ICU admission, yes/no & $20 / 131$ & $9 / 150$ & $0.022^{\mathrm{b}}$ \\
\hline ICU stay, days & $5.2 \pm 1.7$ & $3.8 \pm 1.4$ & $0.040^{\mathrm{b}}$ \\
\hline Mechanical ventilation, yes/no & $19 / 132$ & $9 / 150$ & $0.034^{\mathrm{b}}$ \\
\hline Ventilatory, days & $4.2 \pm 1.6$ & $2.9 \pm 0.9$ & $0.029^{\mathrm{b}}$ \\
\hline Hospital stay, days & $26.2 \pm 21.7$ & $13.3 \pm 10.7$ & $<0.001^{\mathrm{d}}$ \\
\hline Mortality, yes/no & $3 / 148$ & $4 / 155$ & 0.754 \\
\hline
\end{tabular}

${ }^{\mathrm{a}}$ Mean \pm standard deviation or $\mathrm{n} .{ }^{\mathrm{b}} \mathrm{P}<0.05,{ }^{\mathrm{c}} \mathrm{P}<0.01,{ }^{\mathrm{d}} \mathrm{P}, 0.001$. PC, pulmonary contusion; COPD, chronic obstructive pulmonary disease; ISS, injury severity score; $\mathrm{SaO}_{2}$, arterial oxygen saturation; ARDS, acute respiratory distress syndrome; ICU, intensive care unit.

A total of 23 patients had $\mathrm{PC}$ in the right lung, 18 in the left lung and 110 in the bilateral lungs. Except for PC, the other types of chest trauma in the PC group and non-PC group were pneumothorax, haemothorax, haemopneumothorax 


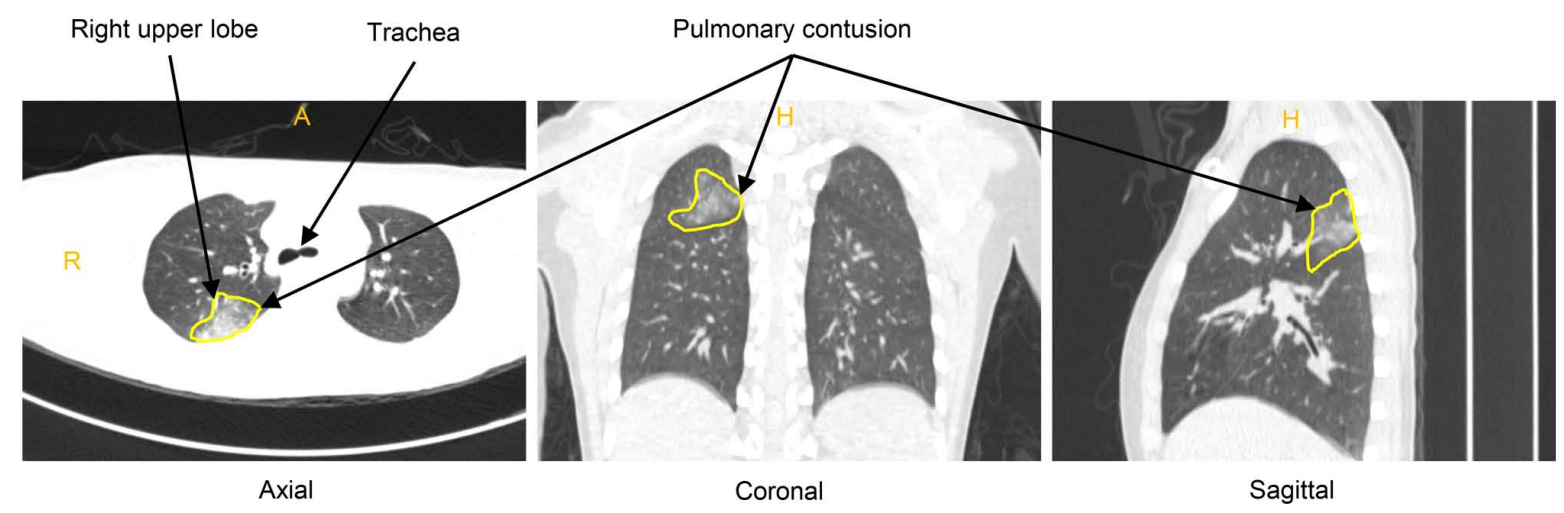

Figure 1. Thoracic computed tomography scan showing pulmonary contusion in the right lung of a patient. A, ahead; R, right; H, head.

and rib fractures. A total of 36 patients in the PC group had $>3$ rib fractures, whilst only 2 patients in the non-PC group had $>3$ rib fractures $(23.8$ vs. $1.3 \%, \mathrm{P}<0.001)$. The ISS was $31.9 \pm 10.0$ for the PC group and $29.9 \pm 9.5$ for the non-PC group $(\mathrm{P}=0.071)$. A total of 136 patients had serious injuries (ISS $>16$ ) in the PC group, whereas 134 patients had serious injuries in the non-PC group $(\mathrm{P}=0.129)$. Significantly more patients had polytrauma in the PC group (129 patients compared with the non-PC group (120 patients; $\mathrm{P}=0.027)$. The arterial oxygen saturation was similar in both groups $(\mathrm{P}=0.324)$ (Table I).

There were 41 patients $(27.2 \%)$ receiving Pleurocan drainage treatment in the PC group and only 13 patients $(8.2 \%)$ in the non-PC group $(\mathrm{P}<0.001)$. Additionally, 49 patients $(32.5 \%)$ had pneumonia complications in the $\mathrm{PC}$ group, whereas 13 patients $(8.2 \%)$ had pneumonia complications in the non-PC group $(\mathrm{P}<0.001)$. Other complications, including pleural effusion and pneumonia with pleural effusion, were similar amongst both groups. A total of 28 patients experienced ARDS in the PC group, whereas 12 patients experienced ARDS in the non-PC group $(\mathrm{P}=0.004)$. Additionally, 20 patients $(13.2 \%)$ were admitted to the intensive care unit (ICU) in the PC group, whereas only 9 patients $(5.7 \%)$ in the non-PC group were admitted $(\mathrm{P}=0.022)$. Patients admitted to the ICU in the PC group stayed in hospital for a longer time period than those in the non-PC group (5.2 \pm 1.8 vs. $3.8 \pm 1.4$ days, respectively; $\mathrm{P}=0.040)$. In the $\mathrm{PC}$ group, 19 patients $(12.6 \%)$ received mechanical ventilation, whereas only 9 patients $(5.7 \%)$ in the non-PC group received mechanical ventilation $(\mathrm{P}=0.034)$. The average ventilatory days for $\mathrm{PC}$ patients were greater than that for non-PC patients $(4.2 \pm 1.6$ vs $2.9 \pm 0.9$ days, respectively; $\mathrm{P}=0.029)$. Overall, $\mathrm{PC}$ patients had a longer hospital stays than non-PC patients $(26.2 \pm 21.7$ vs. $13.3 \pm 10.7$ days, respectively; $\mathrm{P}<0.001)$. However, the mortality rate was similar in both groups (2.0 vs. $2.5 \%$; $\mathrm{P}=0.754$; Table I).

Severity of pulmonary contusion is a risk factor for in-patient outcomes. To determine whether PC volume was associated with in-patient outcomes, the data were analyzed using ROC curves. The area under the curve (AUC) value of 0.828 [standard error, 0.050; 95\% confidence intervals (CI), 0.730-0.927] significantly determined the predictive value of PC volume for the occurrence of ARDS ( $\mathrm{P}<0.001$; Fig. 2A). The AUC value of 0.886 (standard error, $0.040 ; 95 \% \mathrm{CI}$, $0.808-0.964)$ significantly determined the predictive value of PC volume for mechanical ventilation ( $\mathrm{P}<0.001$; Fig. $2 \mathrm{~B})$. The AUC value of 0.793 (standard error, $0.055 ; 95 \% \mathrm{CI}$, 0.684-0.901) significantly determined the predictive value of PC volume for ICU admission ( $\mathrm{P}<0.001$; Fig. 2C). The AUC value of 0.952 (standard error, $0.025 ; 95 \%$ CI, 0.902-1.000) significantly determined the predictive value of $\mathrm{PC}$ volume for mortality ( $\mathrm{P}=0.008$; Fig. 2D).

To further evaluate the impact of PC on in-patient outcomes, binary logistical regression analysis was performed. PC volume was an independent factor influencing the occurrence ratio of ARDS $(\mathrm{P}=0.001)$ and mechanical ventilation $(\mathrm{P}=0.018$; Table II). Furthermore, ARDS was predicted to prolong ICU stay ( $\mathrm{P}=0.010$; Table III). Mechanical ventilation was also predicted to increase the amount of time the patient was required to stay in ICU stay $(\mathrm{P}=0.035)$ and to increase the mortality rate $(\mathrm{P}=0.018$; Table $\mathrm{IV})$.

IL-17 can be used as a biomarker to predict the severity of pulmonary contusion. Several studies have indicated that IL-17 and IL-22 are useful predictive biomarkers in lung diseases (18), infectious diseases $(5)$ and trauma outcomes $(7,8,19)$. However, whether IL-17 and IL-22 are useful biomarkers to predict the severity of PC remains unknown. As expected, IL-17 and IL-22 increased significantly after trauma and peaked on day 5 after trauma. The plasma levels of IL-17 were significantly different between the PC group and the non-PC group on different days after trauma. However, there were no differences in IL-22 between the PC and non-PC groups (Table V). Furthermore, the plasma levels of IL-17 on day 1 or the median of 7 days after trauma were correlated with PC volume (Table VI). Taken together, these data indicate that IL-17 may be used as a biomarker to predict the severity of PC.

IL-17 can be used as a biomarker to predict in-patient outcomes of trauma patients. To determine whether IL-17 was a biomarker for predicting in-hospital outcomes of patients with PC, the relationship between IL-17 and pro-inflammatory complications was assessed, and the predictive value of IL-17 in ARDS, mechanical ventilation, ICU admission and mortality was also determined. High levels of plasma IL-17 were related to a high occurrence ratio of PC. Furthermore, patients with high levels of plasma IL-17 suffered more serious trauma and were more vulnerable to developing ARDS (Table VII). 
A

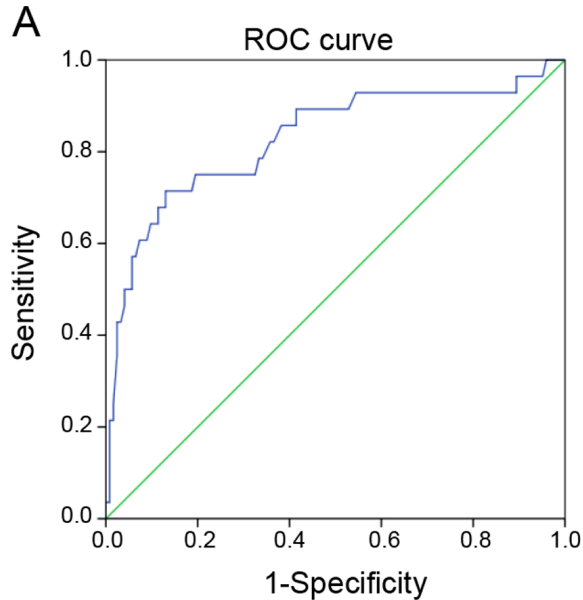

C

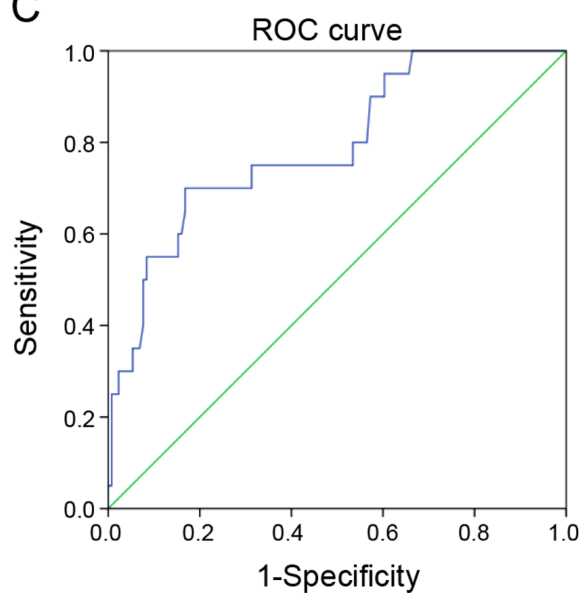

B

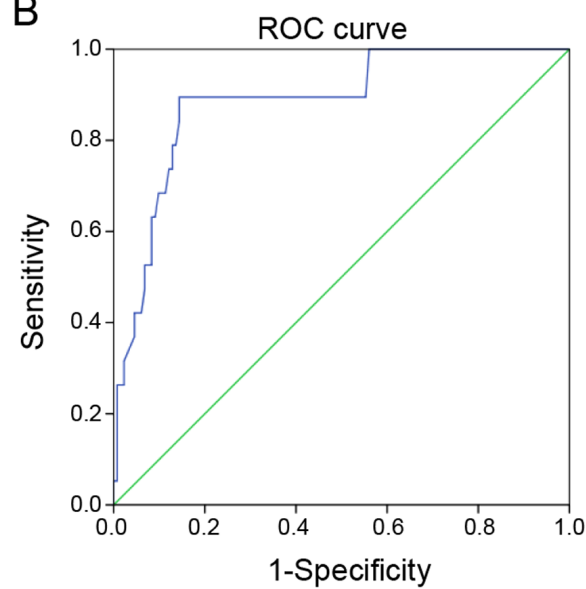

D

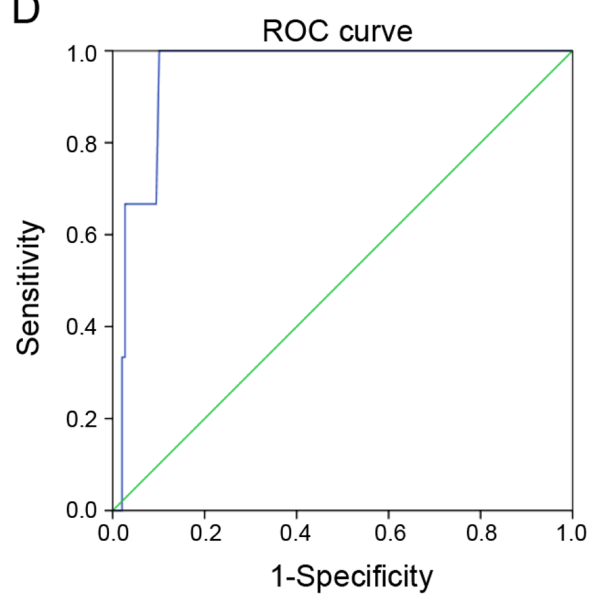

Figure 2. ROC curves of PC volume in predicting in-patient outcomes. (A) ROC curves for PC volume and ARDS. AUC, 0.828; standard error, 0.050; 95\% CI, 0.730-0.927; P<0.001. (B) ROC curves for PC volume and mechanical ventilation. AUC, 0.886; standard error, 0.040; 95\% CI, 0.808-0.964; P<0.001. (C) ROC curves for PC volume and ICU admission. AUC, 0.793; standard error, 0.055; 95\% CI, 0.684-0.901; P<0.001. (D) ROC curves for PC volume and mortality. AUC, 0.828; standard error, 0.025; 95\% CI, 0.902-1.000; P=0.008. ROC, receiver operating characteristic; PC, pulmonary contusion; ARDS, acute respiratory distress syndrome; AUC, area under the curve; CI, confidence interval; ICU, intensive care unit.

ROC analysis was used to calculate the predictive value of IL-17 for in-patient outcomes and found that the levels of plasma IL-17 were useful for predicting ARDS occurrence, mechanical ventilation, ICU admission and mortality (Fig. 3).

\section{Discussion}

$\mathrm{PC}$ is very common in patients who suffer blunt chest traumas, and the severity of PC ranges from mild to life-threatening $(3,20)$. In the present study, the incidence of blunt chest trauma was $1.9 \%$, and the incidence of PC in these patients was $34.5 \%$, which was similar to previous studies (1-3). The major cause of injury was motor vehicle crashes, which was also the major cause of PC. This was primarily because in motor vehicle crashes, patients are hit in the chest with a powerful impact, and the chest wall is compressed and then rebounds. This was hypothesized to be the primary cause of PC $(1,21)$. Other causes, including injuries related to bicycles, walking, falling and explosions, were similar in the $\mathrm{PC}$ group and the non-PC group. The baseline data of participants, which included age, sex and comorbidities, were similar in the $\mathrm{PC}$ and non-PC groups. Thus, these cohorts were suitable for comparing between the PC group and the non-PC group.
Isolated PC is rare, and PC is often accompanied by serious bony thorax damage, such as rib fractures (22). In the present study, the majority of the PC was located in both the lungs. PC patients had a high incidence of other types of chest traumas, including pneumothorax, haemothorax, haemopneumothorax and rib fractures. Furthermore, patients with $>3$ rib fractures were more likely to experience PC. Previous studies have shown that the number of rib fractures was a predictor of the severity of PC (22), and the number of fractured ribs best predicted the appearance of complications (such as delayed pleuropulmonary complications and increased bleeding) (23). In the present study, it was also shown that patients with PC had a higher rate of polytraumas. A possible explanation for this was that patients with more rib fractures or polytraumas had higher-energy impacts, which were always accompanied by blunt chest trauma.

Several studies have shown that PC patients have high incidences of negative pulmonary outcomes $(4,22)$. The present study showed that patients with PC were more likely to develop ARDS, were more likely to be admitted to the ICU and receive mechanical ventilation. Patients with PC had longer periods receiving assisted ventilation, ICU stays and total length of hospital stays. These findings were consistent with those of a previous study (4). However, there was no difference 
Table II. Logistical regression analysis of variables associated with pulmonary contusion outcomes.

\begin{tabular}{|c|c|c|c|c|c|c|}
\hline \multirow[b]{2}{*}{ Clinicopathological characteristic } & \multirow[b]{2}{*}{$\beta$} & \multirow[b]{2}{*}{ Standard error } & \multirow[b]{2}{*}{ P-value } & \multirow[b]{2}{*}{$\operatorname{Exp}(\beta)$} & \multicolumn{2}{|c|}{$\begin{array}{l}95 \% \text { confidence } \\
\text { interval for } \operatorname{Exp}(\beta)\end{array}$} \\
\hline & & & & & Lower & Upper \\
\hline \multicolumn{7}{|l|}{ Mortality } \\
\hline PC volume & -0.664 & 0.396 & 0.094 & 0.515 & 0.237 & 1.120 \\
\hline \multicolumn{7}{|l|}{ Mechanical ventilation } \\
\hline Rib fractures & -5.439 & 2.029 & 0.007 & 0.004 & 0.000 & 0.232 \\
\hline Blood products & 2.863 & 1.163 & 0.014 & 17.514 & 1.792 & 171.158 \\
\hline PC volume & -0.283 & 0.119 & 0.018 & 0.753 & 0.596 & 0.952 \\
\hline ARDS & 3.263 & 1.191 & 0.006 & 26.127 & 2.531 & 269.701 \\
\hline \multicolumn{7}{|l|}{ ARDS } \\
\hline $\mathrm{SaO}_{2}$ & 0.504 & 0.137 & $<0.001$ & 1.655 & 1.266 & 2.164 \\
\hline PC volume & -0.124 & 0.036 & 0.001 & 0.883 & 0.823 & 0.948 \\
\hline
\end{tabular}

PC, pulmonary contusion; ARDS, acute respiratory distress syndrome; $\mathrm{SaO}_{2}$, arterial oxygen saturation.

Table III. Relationship between ARDS and patient outcomes.

\begin{tabular}{lccc}
\hline Variables & ARDS $^{\mathrm{a}}$ & Non-ARDS $^{\mathrm{a}}$ & P-value \\
\hline ICU stay, days, & $5.7 \pm 1.9$ & $3.8 \pm 1.3$ & $0.010^{\mathrm{b}}$ \\
Hospital stay, days & $18.9 \pm 9.5$ & $19.7 \pm 8.0$ & 0.792 \\
Mortality, yes/no & $2 / 38$ & $5 / 265$ & 0.224
\end{tabular}

${ }^{\mathrm{a}}$ Mean \pm standard deviation or $\mathrm{n} ;{ }^{\mathrm{b}} \mathrm{P}<0.05$. ARDS, acute respiratory distress syndrome; ICU, intensive care unit.

Table IV. Relationship between mechanical ventilation and patient outcomes.

\begin{tabular}{lccc}
\hline Variables & Ventilation $^{\mathrm{a}}$ & No-Ventilation $^{\mathrm{a}}$ & P-value \\
\hline ICU stay, days & $5.4 \pm 2.0$ & $3.4 \pm 0.5$ & $0.035^{\mathrm{b}}$ \\
Hospital stay, days & $18.6 \pm 7.4$ & $19.7 \pm 8.2$ & 0.763 \\
Mortality, yes/no & $3 / 25$ & $4 / 278$ & $0.018^{\mathrm{b}}$ \\
\hline
\end{tabular}

${ }^{\mathrm{a}}$ Mean \pm standard deviation or $\mathrm{n} ;{ }^{\mathrm{b}} \mathrm{P}<0.05$. ICU, intensive care unit.

in mortality between the PC group and the non-PC group. A possible explanation for this is that mortality was related to several factors, and PC usually affects the pulmonary situation, which recovers very well after effective supportive care.

$\mathrm{PC}$ volume represents the size of contused lung, and a previous study showed that quantifying PC volume could allow identification of patients at high risk of ARDS and that CT contusion volume was significantly correlated with injury severity in patients with blunt chest trauma (17). In the present study, PC volume was significantly associated with ARDS occurrence, mechanical ventilation rate, ICU admission and mortality. Furthermore, in the logistical regression analysis, PC volume was an independent predictive factor for ARDS occurrence and mechanical ventilation rate. The data also showed that ARDS and mechanical ventilation could prolong ICU stay. Mortality rates were also higher amongst patients receiving mechanical ventilation. Thus, these findings support the hypothesis that $\mathrm{PC}$ volume was effective in predicting the outcomes of PC patients.

IL-17 is a glycosylated protein that is associated with the severity of inflammation in tissues by inducing the production of other pro-inflammatory mediators, including IL-1, TNF, IL-6, IL-8, CCL20 and G-CSF, which collectively results in an influx of neutrophils (24). IL-17 exhibits synergistic function with other mediators, including IL-1, IL- 6 and TNF- $\alpha$, to activate tissue-infiltrating neutrophils, and these neutrophils facilitate the effective elimination of invading bacteria or fungi (25). Previous studies have shown that IL-17 serves a central role in the pathogenesis of the systemic inflammatory response to injury (26). A high plasma IL-17 concentration has a unique and independent association with susceptibility to sepsis in polytrauma patients (7). In the present study, IL-17 and IL-22 levels were increased significantly after PC and peaked on day 5 after PC diagnosis. However, only the plasma IL-17 levels were significantly different between the PC group and the non-PC group. Furthermore, both plasma IL-17 levels on the initial day and median levels of IL-17 on day 7 were strongly associated with PC volume. The results indicated that IL-17 was a biomarker for predicting the severity of PC. Dai et al (27) found that cytokines (IL-17) and an imbalance of Treg/Th17 were significantly relevant to tissue (lung and intestine) inflammation and injury in the acute phase (within $8 \mathrm{~h}$ ) after multiple traumas. This group also found that IL-17 was crucially involved in the pathogenesis of multiple traumas in rats, and IL-17 inhibition might ameliorate lung inflammation in the acute phase after multiple traumas (8). The potential explanation was that IL-17 might induce production of other pro-inflammatory cytokines and increase the uncontrolled inflammatory response induced by high-energy traumas, which may be the primary cause of lung impairment. 
Table V. Plasma levels of IL-17 and IL-22 in the patients with or without PC.

\begin{tabular}{|c|c|c|c|c|}
\hline Cytokines & Control & $\mathrm{PC}$ group, $\mathrm{n}=151$ & Non-PC group, $n=159$ & P-value \\
\hline IL-17, pg/ml (range) & $32.1 \pm 11.2(0-45)$ & & & $<0.001^{\mathrm{a}, \mathrm{b}}$ \\
\hline Day 1 & & $179.5 \pm 167.7^{\mathrm{a}}$ & $95.0 \pm 81.6^{\mathrm{b}}$ & $<0.001$ \\
\hline Day 3 & & $330.2 \pm 244.8$ & $212.6 \pm 175.2$ & $<0.001$ \\
\hline Day 5 & & $535.7 \pm 285.3$ & $321.9 \pm 218.4$ & $<0.001$ \\
\hline Day 7 & & $267.3 \pm 218.1$ & $193.5 \pm 171.0$ & 0.001 \\
\hline IL-22, pg/ml (range) & $33.7 \pm 9.3(0-53.3)$ & & & $<0.001^{\mathrm{c}, \mathrm{d}}$ \\
\hline Day 1 & & $62.9 \pm 44.9^{c}$ & $62.3 \pm 41.3^{\mathrm{d}}$ & 0.902 \\
\hline Day 3 & & $127.5 \pm 85.9$ & $126.1 \pm 77.4$ & 0.878 \\
\hline Day 5 & & $222.9 \pm 130.2$ & $239.6 \pm 125.4$ & 0.25 \\
\hline Day 7 & & $89.8 \pm 51.0$ & $97.1 \pm 52.0$ & 0.216 \\
\hline
\end{tabular}

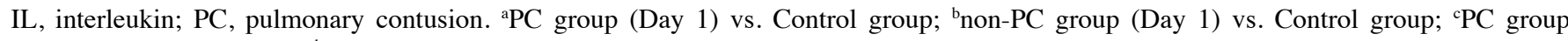
(Day 1) vs. Control group; ${ }^{\mathrm{d}}$ non-PC group (Day 1) vs. Control group.

Table VI. Relationship between plasma levels of IL-17 and PC volume.

\begin{tabular}{lcc}
\hline Statistical parameter & IL-17 (Day 1) and PC volume & IL-17 (median) and PC volume \\
\hline Spearman's rho correlation coefficient & 0.922 & 0.923 \\
P, two-tailed & $<0.001^{\mathrm{a}}$ & $<0.001^{\mathrm{a}}$ \\
$\mathrm{n}$ & 151 & 151 \\
\hline
\end{tabular}

${ }^{\mathrm{a}} \mathrm{P}<0.05$. IL, interleukin; PC, pulmonary contusion; IL-17 (Day 1), plasma level of IL-17 on the day when patients were admitted to the hospital; IL-17 (Median), plasma level of IL-17 on all 7 days after trauma.

Table VII. Relationship between IL-17 and pro-inflammatory complications.

\begin{tabular}{|c|c|c|c|c|c|c|}
\hline \multirow[b]{2}{*}{ Complications } & \multicolumn{2}{|c|}{ IL-17, Day $1^{\mathrm{a}}$} & \multirow[b]{2}{*}{ P-value } & \multicolumn{2}{|c|}{ IL-17, median ${ }^{\mathrm{a}}$} & \multirow[b]{2}{*}{ P-value } \\
\hline & $\leq 45 \mathrm{pg} / \mathrm{ml}$ & $>45 \mathrm{pg} / \mathrm{ml}$ & & $\leq 90 \mathrm{pg} / \mathrm{ml}$ & $>90 \mathrm{pg} / \mathrm{ml}$ & \\
\hline $\mathrm{PC}$ & & & 0.009 & & & $<0.001^{\mathrm{c}}$ \\
\hline $\mathrm{PC}$ & 28 & 123 & & 29 & 122 & \\
\hline Non-PC & 50 & 109 & & 61 & 98 & \\
\hline ISS & $23.4 \pm 10.6$ & $29.1 \pm 11.1$ & $<0.001$ & $23.8 \pm 10.5$ & $29.2 \pm 11.2$ & $<0.001^{\mathrm{c}}$ \\
\hline ARDS & & & 0.018 & & & $0.036^{\mathrm{b}}$ \\
\hline ARDS & 4 & 36 & & 6 & 34 & \\
\hline Non-ARDS & 74 & 196 & & 84 & 186 & \\
\hline
\end{tabular}

${ }^{\mathrm{a}}$ Mean \pm standard deviation or $\mathrm{n} ;{ }^{\mathrm{b}} \mathrm{P}<0.05 ;{ }^{\mathrm{C}} \mathrm{P}<0.001$. IL, interleukin; $\mathrm{PC}$, pulmonary contusion; ISS, injury severity score; ARDS, acute respiratory distress syndrome; IL-17 Day 1, plasma level of IL-17 on the day when patients were admitted to the hospital; IL-17 median, plasma level of IL-17 on all 7 days after trauma.

Given that PC volume was effective in predicting the outcomes of $\mathrm{PC}$ patients and that IL-17 had a strong relationship with PC volume, whether IL-17 could be used as a biomarker to predict in-patient outcomes of patients with PC was next assessed. High levels of plasma IL-17 were associated with a high occurrence ratio of PC. Furthermore, patients with a high level of plasma IL-17 suffered a more serious trauma and were more vulnerable to developing ARDS. Using ROC analysis to calculate the predictive value of IL-17 for the in-patient outcomes showed that the levels of plasma IL-17 were useful for predicting the occurrence of ARDS, mechanical ventilation, ICU admission and mortality. These results suggest that IL-17 was significantly associated with pro-inflammatory complications in serious trauma patients and could be used as 
A
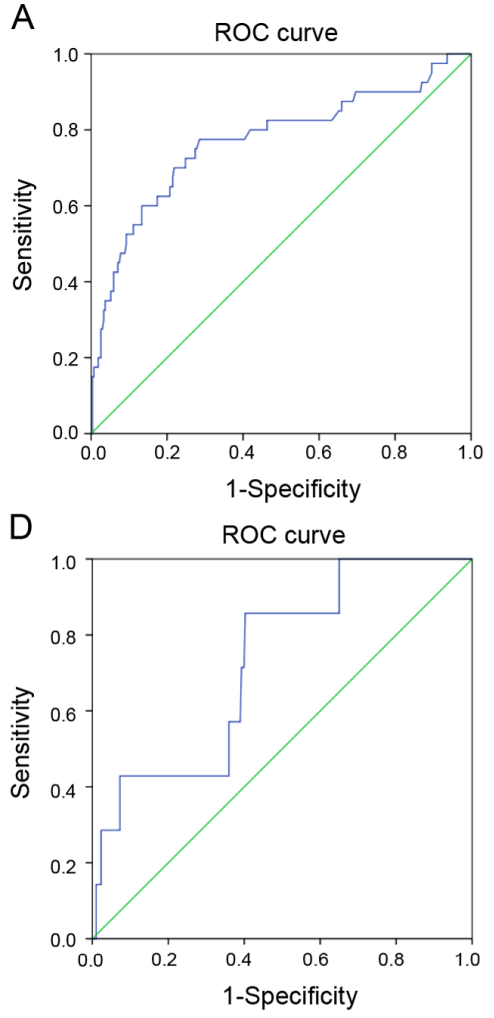

G

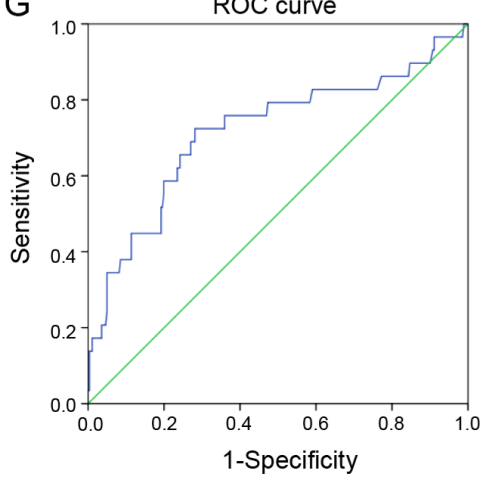

B

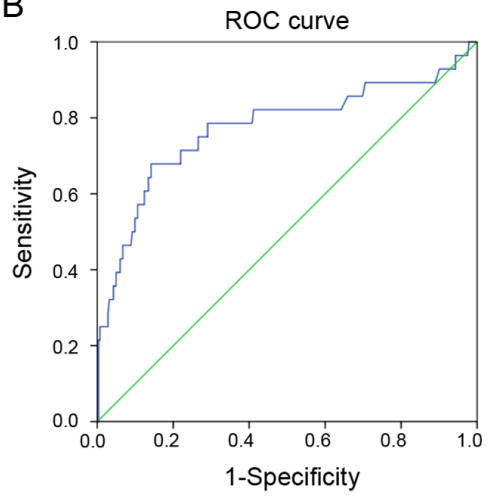

E

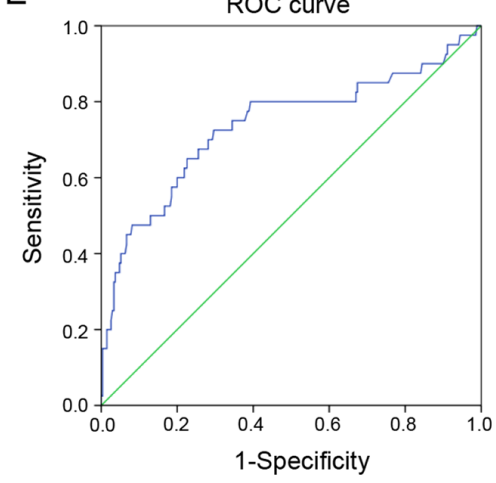

$\mathrm{H}$

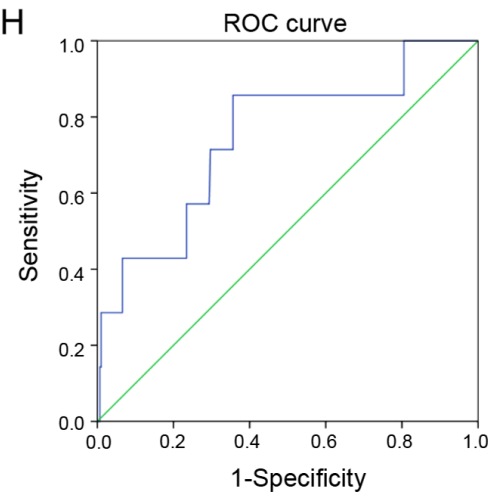

C

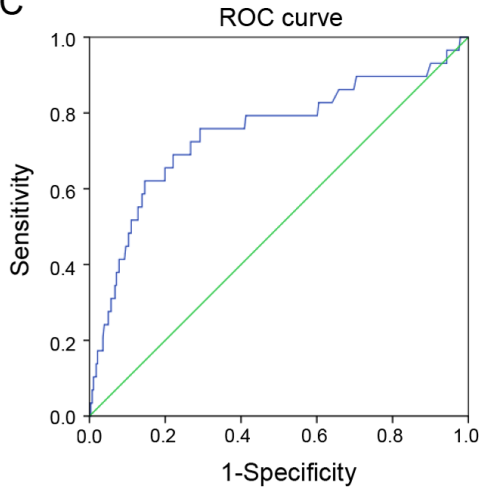

$\mathrm{F}$

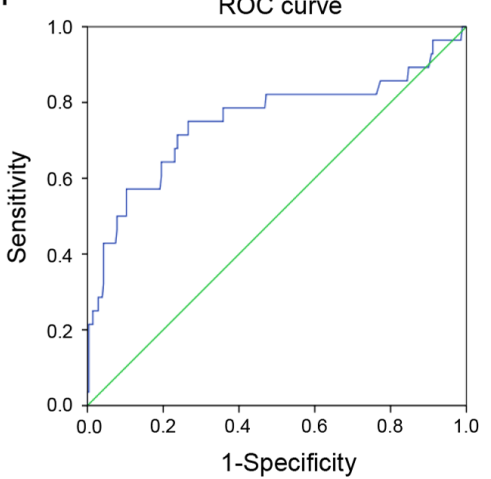

Figure 3. ROC curves of IL-17 for predicting the in-hospital outcomes of patients. (A) ROC curves for IL-17 (day 1) and ARDS. AUC, 0.770; standard error, 0.047; 95\% CI, 0.679-0.862; P<0.001. (B) ROC curves for IL-17 (day 1) and mechanical ventilation. AUC, 0.773; standard error, 0.058; 95\% CI, 0.659-0.887; $\mathrm{P}<0.001$. (C) ROC curves for IL-17 (day 1) and ICU admission. AUC 0.747; standard error, 0.057; 95\% CI, 0.636-0.858; P<0.001. (D) ROC curves for IL-17 (day 1) and mortality. AUC, 0.727; standard error, 0.086; 95\% CI, 0.558-0.897; P=0.040. (E) ROC curves for IL-17 (median) and ARDS. AUC, 0.737; standard error, 0.051; 95\% CI, 0.638-0.837; P<0.001. (F) ROC curves for IL-17 (median) and mechanical ventilation, AUC, 0.752; standard error, 0.061; 95\% CI, 0.632-0.871; P<0.001. (G) ROC curves for IL-17 (median) and ICU admission. AUC, 0.717; standard error, 0.059; 95\% CI, 0.602-0.832; P<0.001. (H) ROC curves for IL-17 (median) and mortality. AUC, 0.747; standard error, 0.099; 95\% CI, 0.552-0.941; P=0.026. ROC, receiver operating characteristic; IL, interleukin; ARDS, acute respiratory distress syndrome; AUC, area under the curve; CI, confidence interval; ICU, intensive care unit.

a biomarker to predict in-patient outcomes of patients with PC. However, whether increased levels of IL-17 was due to polytrauma or the PC was not determined. However, PC seemed to increase the levels of IL-17. To the best of our knowledge, this is the first time that IL-17 has been associated with the severity of $\mathrm{PC}$ and the outcomes of $\mathrm{PC}$ patients, though the underlying mechanisms require further clarification.

IL-22 is a homeostatic cytokine with pronounced tissue-protective properties (10). It was found to preserve the integrity of boundary organs and tissues (28), and serves an important role in the resolution of sterile and nonsterile inflammation, organ injury, wound healing and regeneration $(29,30)$. IL-22 is also associated with burn injuries and sepsis $(14,15)$. In the present study, IL-22 increased significantly following
PC and peaked on day 5 after PC diagnosis. However, there was no association between IL-22 and PC outcomes. A potential explanation for this is that IL-22 may not be a specific cytokine for PC during multiple traumas. It may be secreted at various sites during serious trauma. This is different from lung injuries which are induced by burns or infection $(14,15)$. Further studies are required to determine the specific role of IL-22 in multiple traumas.

In summary, the present study showed that $\mathrm{PC}$ volume was effective in predicting the outcomes of PC patients. IL-17 could be used as a biomarker to predict the severity of PC and was strongly associated with PC volume. IL-17 was significantly associated with pro-inflammatory complications in PC patients and could be used as a biomarker to 
predict outcomes of PC in-patients. IL-17 is thus a potential biomarker for predicting the severity and outcomes of PC in trauma patients. However, whether elevated plasma IL-17 concentrations can be used as a marker of other processes of post-trauma, and whether it is a causal factor for PC remains unknown. Further studies are thus required to explore the mechanisms.

\section{Acknowledgements}

Not applicable.

\section{Funding}

This work was supported by Talents Sub-Highland of Emergency and Medical Rescue of Guangxi Province in China (grant no. GXJZ201405), Health Commission of Guangxi (grant no. Z2016289), the Young Teachers' Basic Ability Improvement in Guangxi University Project (grant no. 2019KY0108) and the Medical Excellence Award funded by the Creative Research Development Grant from the First Affiliated Hospital of Guangxi Medical University.

\section{Availability of data and materials}

All data generated and/or analyzed during the present study are included in this published article. Raw data is available from the corresponding authors upon reasonable request and with the permission of the First Affiliated Hospital of Guangxi Medical University.

\section{Authors' contributions}

SL analyzed the clinical data, performed the experiments and wrote the original draft. QQ, DL, WP, YW and YX collected the patient samples and revised the manuscript. JW and XY collected the clinical data. JZ and LS designed the study, and reviewed and edited the manuscript. All authors read and approved the final manuscript.

\section{Ethics approval and consent to participate}

Written informed consent was obtained from each participant prior to enrolment, and this study was approved by the Medical Ethics Committee of The First Affiliated Hospital of Guangxi Medical University.

\section{Patient consent for publication}

Not applicable.

\section{Competing interests}

The authors declare that they have no competing interests.

\section{References}

1. Cohn SM and Dubose JJ: Pulmonary contusion: An update on recent advances in clinical management. World J Surg 34: 1959-1970, 2010
2. Ganie FA, Lone H, Lone GN, Wani ML, Singh S, Dar AM, Wani NU, Wani SN and Nazeer NU: Lung contusion: A clinico-pathological entity with unpredictable clinical course. Bull Emerg Trauma 1: 7-16, 2013.

3. Rodriguez RM, Friedman B, Langdorf MI, Baumann BM, Nishijima DK, Hendey GW, Medak AJ, Raja AS and Mower WR: Pulmonary contusion in the pan-scan era. Injury 47: 1031-1034, 2016.

4. Prunet B, Bourenne J, David JS, Bouzat P, Boutonnet M, Cordier PY, Renaudin P, Meaudre E and Michelet P: Patterns of invasive mechanical ventilation in patients with severe blunt chest trauma and lung contusion: A French multicentric evaluation of practices. J Intensive Care Soc 20: 46-52, 2019.

5. Valeri M and Raffatellu M: Cytokines IL-17 and IL-22 in the host response to infection. Pathog Dis 74: ftw111, 2016.

6. Becker KL, Snider R and Nylen ES: Procalcitonin assay in systemic inflammation, infection, and sepsis: Clinical utility and limitations. Crit Care Med 36: 941-952, 2008.

7. Ahmed Ali M, Mikhael ES, Abdelkader A, Mansour L, El Essawy R,El Sayed R,Eladawy A and Mukhtar A: Interleukin-17 as a predictor of sepsis in polytrauma patients: A prospective cohort study. Eur J Trauma Emerg Surg 44: 621-626, 2018.

8. Dai H, Xu L, Tang Y, Liu Z and Sun T: Treatment with a neutralising anti-rat interleukin-17 antibody after multiple-trauma reduces lung inflammation. Injury 46: 1465-1470, 2015.

9. Mizoguchi A, Yano A, Himuro H, Ezaki Y, Sadanaga T and Mizoguchi E: Clinical importance of IL-22 cascade in IBD. J Gastroenterol 53: 465-474, 2018.

10. Mühl H, Scheiermann P, Bachmann M, Härdle L, Heinrichs A and Pfeilschifter J: IL-22 in tissue-protective therapy. Br J Pharmacol 169: 761-771, 2013.

11. Alcorn JF: IL-22 plays a critical role in maintaining epithelial integrity during pulmonary infection. Front Immunol 11: 1160, 2020.

12. Hoegl S, Bachmann M, Scheiermann P, Goren I, Hofstetter C, Pfeilschifter J, Zwissler B and Muhl H: Protective properties of inhaled IL-22 in a model of ventilator-induced lung injury. Am J Respir Cell Mol Biol 44: 369-376, 2011

13. McAleer JP and Kolls JK: Directing traffic: IL-17 and IL-22 coordinate pulmonary immune defense. Immunol Rev 260: 129-144, 2014.

14. Rendon JL and Choudhry MA: Th17 cells: Critical mediators of host responses to burn injury and sepsis. J Leukoc Biol 92: $529-538,2012$.

15. Li G, Zhang L, Han N, Zhang K and Li H: Increased Th17 and Th22 cell percentages predict acute lung injury in patients with sepsis. Lung 198: 687-693, 2020.

16. Baker SP, O'Neill B, Haddon W Jr and Long WB: The injury severity score: A method for describing patients with multiple injuries and evaluating emergency care. J Trauma 14: 187-196, 1974.

17. Mahmood I, El-Menyar A, Younis B, Ahmed K, Nabir S, Ahmed MN, Al-Yahri O, Mahmood S, Consunji R and Al-Thani H: Clinical significance and prognostic implications of quantifying pulmonary contusion volume in patients with blunt chest trauma. Med Sci Monit 23: 3641-3648, 2017.

18. Tufman A, Huber RM, Völk S, Aigner F, Edelmann M, Gamarra F, Kiefl R, Kahnert K, Tian F, Boulesteix AL, et al: Interleukin-22 is elevated in lavage from patients with lung cancer and other pulmonary diseases. BMC Cancer 16: 409, 2016.

19. Abboud A, Namas RA, Ramadan M, Mi Q, Almahmoud K, Abdul-Malak O, Azhar N, Zaaqoq A, Namas R, Barclay DA, et al: Computational analysis supports an early, type 17 cell-associated divergence of blunt trauma survival and mortality. Crit Care Med 44: e1074-e1081, 2016.

20. Pehlivanlar Küçük M, Küçük AO, Aksoy İ, Aydın D and Ülger F: Prognostic evaluation of cases with thoracic trauma admitted to the intensive care unit: 10-year clinical outcomes. Ulus Travma Acil Cerrahi Derg 25: 46-54, 2019.

21. Požgain Z, Kristek D, Lovrić I, Kondža G, Jelavić M, Kocur J and Danilović M: Pulmonary contusions after blunt chest trauma: Clinical significance and evaluation of patient management. Eur J Trauma Emerg Surg 44: 773-777, 2018.

22. Miller C, Stolarski A, Ata A, Pfaff A, Nadendla P, Owens K, Evans L, Bonville D, Rosati C, Stain SC and Tafen M: Impact of blunt pulmonary contusion in polytrauma patients with rib fractures. Am J Surg 218: 51-55, 2019.

23. Flores-Funes D, Lluna-Llorens AD, Jiménez-Ballester MÁ, Valero-Navarro G, Carrillo-Alcaráz A, Campillo-Soto Á and Aguayo-Albasini JL: Is the number of rib fractures a risk factor for delayed complications? A case-control study. Eur J Trauma Emerg Surg 46: 435-440, 2020. 
24. Dominguez-Villar $M$ and Hafler DA: Immunology. An innate role for IL-17. Science 332: 47-48, 2011.

25. Schwarzenberger P, Huang W, Ye P, Oliver P, Manuel M, Zhang Z, Bagby G, Nelson S and Kolls JK: Requirement of endogenous stem cell factor and granulocyte-colony-stimulating factor for IL-17-mediated granulopoiesis. J Immunol 164: 4783-4789, 2000.

26. Frangen TM, Bogdanski D, Schinkel C, Roetman B, Kälicke T, Muhr G and Köller M: Systemic IL-17 after severe injuries. Shock 29: 462-467, 2008

27. Dai H, Sun T, Liu Z,Zhang J and Zhou M: The imbalance between regulatory and IL-17-secreting $\mathrm{CD} 4^{+} \mathrm{T}$ cells in multiple-trauma rat. Injury 44: 1521-1527, 2013.
28. Eyerich K, Dimartino V and Cavani A: IL-17 and IL-22 in immunity: Driving protection and pathology. Eur J Immunol 47: 607-614, 2017.

29. Alabbas SY, Begun J, Florin TH and Oancea I: The role of IL-22 in the resolution of sterile and nonsterile inflammation. Clin Transl Immunology 7: e1017, 2018.

30. Brockmann L, Giannou AD, Gagliani N and Huber S: Regulation of TH17 cells and associated cytokines in wound healing, tissue regeneration, and carcinogenesis. Int J Mol Sci 18: 1033, 2017.

This work is licensed under a Creative Commons Attribution-NonCommercial-NoDerivatives 4.0 International (CC BY-NC-ND 4.0) License. 